\title{
BMJ Open Access to mental healthcare in the year after first fit note: a longitudinal study of linked clinical records
}

\author{
Sarah Dorrington (D) , ${ }^{1,2}$ Ewan Carr (D) , ${ }^{1}$ Sharon Stevelink, ${ }^{1}$ Mark Ashworth (D) , ${ }^{3}$ \\ Matthew Broadbent, ${ }^{2}$ Ira Madan, ${ }^{4}$ Stephani Hatch, ${ }^{1}$ Matthew Hotopf ${ }^{1,2}$
}

To cite: Dorrington S, Carr E, Stevelink S, et al. Access to mental healthcare in the year after first fit note: a longitudinal study of linked clinical records. BMJ Open 2021;11:e044725. doi:10.1136/ bmjopen-2020-044725

- Prepublication history and additional supplemental material for this paper are available online. To view these files, please visit the journal online (http://dx.doi.org/10.1136/ bmjopen-2020-044725).

Received 12 September 2020 Accepted 12 July 2021

Check for updates

(c) Author(s) (or their employer(s)) 2021. Re-use permitted under CC BY-NC. No commercial re-use. See rights and permissions. Published by BMJ.

${ }^{1}$ Department of Psychological Medicine, Institute of Psychiatry Psychology and Neuroscience, King's College London, London, UK

${ }^{2}$ National Institute for Health Research Maudsley Biomedical Research Centre, South London and Maudsley NHS Foundation Trust, London, UK

${ }^{3}$ School of Population Health \& Environmental Sciences, King's College London, London, UK

${ }^{4}$ Department of Occupational Health, Guy's and St Thomas' Hospitals NHS Trust, London, UK

Correspondence to

Dr Sarah Dorrington; sarah.dorrington@kcl.ac.uk

\section{ABSTRACT}

Objectives Sickness absence is strongly associated with poor mental health, and mental disorders often go untreated. In this population-based cohort study, we identified people receiving fit notes from their general practitioner (GP) and determined access to mental health treatment stratified by health complaint and demographic variables.

Design Longitudinal study of health records.

Setting Primary care and secondary mental health care in the borough of Lambeth, South London. Forty-five GP practices in Lambeth and the local secondary mental healthcare trust.

Participants The analytical sample included 293933 working age adults (16-60 years) registered at a Lambeth GP practice between 1 January 2014 and 30 April 2016.

Primary and secondary outcome measures Three indicators of mental healthcare in the year after first fit note were antidepressant prescription, contact with Improving Access to Psychological Therapy (IAPT) services and contact with secondary mental health services. Results $75 \%$ of people with an identified mental health condition at first fit note had an indicator of mental healthcare in the following year. Black Caribbean and Black African groups presenting with mental disorders were less likely to have a mental healthcare indicator compared with White British groups.

Conclusions The majority of those with an identified mental health need receive some treatment in the year following a fit note; however, our results suggest Black African and Black Caribbean groups with an identified mental healthcare need have less complete access compared to the White British group.

\section{INTRODUCTION}

Mental and behavioural conditions have risen in the past three decades to become the leading cause of certification for long-term sickness absence (LTSA) in the UK. ${ }^{1-3}$ In $2017 / 2018$, LTSA is estimated to have cost $£ 15$ billion to the UK economy. ${ }^{4}$ A large proportion of LTSA is attributable to musculoskeletal disorders and mental disorders, chronic conditions that are common throughout adult life and that are associated with high disability. ${ }^{56} \mathrm{~A}$ critical question is whether there are existing treatment

\section{Strengths and limitations of this study}

- Working with digital health records provided information on virtually all individuals seeking help within the primary care population.

- We focused on a single geographical area for which we had GP data; this is not representative of other populations, and our findings should be tested in other areas.

- We were unable to explore contextual factors such as the nature of employment, educational level or benefit information.

- Long-term health conditions were assessed using the Quality and Outcomes Framework, which provides incomplete diagnostic information.

- Our mental health indicators were limited to antidepressants, IAPT and secondary care contact; therefore, our estimates of treatment are likely to be an underestimate.

gaps that could be targeted through early intervention to prevent conditions leading to occupational impairment and LTSA. ${ }^{7}$

Presentation to the general practitioner (GP) for sickness certification is a key point in the development of LTSA. In the UK, sickness absence beyond 7 days requires certification, most often delivered by a GP. Presentation for a fit note is an opportunity for intervention, an insight that led to a change in policy in 2010, when the 'fit note' replaced the sick note. The fit note was designed as an opportunity to promote early access to treatment. In addition, instead of either being 'fit' or 'not fit' for work, the fit note introduced the option of being 'maybe fit'. GPs are invited to recommend work adjustments on the fit note to enable patients to remain in work or return to work. ${ }^{\text {? }}$

A third of fit notes are for mental health conditions, yet common mental disorders frequently go undiagnosed and undertreated. ${ }^{8-10}$ The Adult Psychiatric Morbidity Survey 2014 showed $61 \%$ adults in England aged 16-74 years with conditions such as depression and anxiety do not access mental health treatment. ${ }^{11}$ For the 
$39 \%$ of patients surveyed who do receive treatment, the majority are managed in primary care, which includes antidepressant use and referral for brief psychological interventions including Improved Access to Psychological Treatment (IAPT) services. Patients with treatment resistant, complex and more severe conditions tend to have contact with secondary care, which includes community mental health team input and psychiatric admissions.

We have previously shown, using data from South London, that fit note receipt is highest among groups with a high prevalence of morbidity: women, older age groups, Black minority ethnic groups and people with higher levels of deprivation. ${ }^{12}{ }^{13}$ In this paper, we explore the extent to which people receiving fit notes access mental health treatment across primary and secondary care and what scope there is to improve access to care and thereby health and occupational outcomes. First, we analyse access to mental health treatment in the population in the year after their first fit note by demographic factors. Second, we analyse variation in access to mental health treatment by condition recorded by the GP at the consultation for the first fit note, and lastly, we analyse demographic variation in no contact with mental healthcare among people with a mental health or stress condition at first fit note. To interpret demographic patterns of mental healthcare service use in people who had fit notes, we include a baseline analysis of mental health treatment use in the whole working age population for comparison with the population receiving a fit note.

\section{METHODS}

\section{Study population}

The study is based in the London Borough of Lambeth, home to a population of 327582 people $^{14}$ and characterised by high deprivation, considerable income inequality and a high proportion of the population $(38 \%)$ born outside of the UK. ${ }^{15} 16$ The population is young and ethnically diverse, including large populations of people who identify as Black African, Black Caribbean and Black British, as well as South American and Portuguese.

\section{Data sources}

Primary care source data: Lambeth DataNet (LDN)

LDN is a primary care database of patients registered at all 45 general practices within the London Borough of Lambeth. LDN includes patient-level data on demographics, clinical diagnoses, referrals, consultations, prescriptions, laboratory tests and public health initiatives, including the Quality and Outcomes Framework (QOF) data. The database has been described in detail in a previous publication. ${ }^{12}$

\section{Secondary care source data: Clinical Record Interactive Search (CRIS)}

The South London and Maudsley (SLaM) National Health Service (NHS) Foundation Trust provides comprehensive mental healthcare for residents of the London Borough of Lambeth. The electronic health records are interrogated for research purposes with the CRIS system, which deidentifies the entire health record. ${ }^{17}$ The IAPT programme, which provides primary care psychological treatment for common mental disorders, is provided by SLaM alongside secondary care services and is part of CRIS.

\section{Measures}

Demographic variables (LDN)

Information on individuals' first recorded gender, year of birth, ethnicity and 2011-defined lower super output area (LSOA; an area covering an average population of approximately 1700 individuals $)^{18}$ were extracted from LDN. Index of Multiple Deprivation (IMD) is the official measure of relative deprivation for small areas in England. LSOA of residence was used to derive a measure of deprivation for each individual based on the IMD (IMD 2010, after conversion to 2011 LSOA boundaries). IMD scores were divided into quintiles, based on deprivation scores in Lambeth. Ethnicity was coded using eight subcategories of self-identified ethnicity (White, White Other,Black African, Asian, Black Caribbean, Mixed, Other and Black Other).

\section{Long-term health conditions and pain (LDN)}

Presence of long-term health conditions was assessed using QOF data from LDN. QOF is an annual reward and incentive programme for all GP practices in England. It requires GPs to identify individuals who meet QOF targets for various long-term conditions such as depression, obesity and diabetes. We included in our analysis a count of long-term conditions defined using $15 \mathrm{QOF}$ definitions ${ }^{19}$ : depression, epilepsy, diabetes mellitus (DM), coronary heart disease (CHD), chronic obstructive pulmonary disorder (COPD), cancer (non-specified), atrial fibrillation $(\mathrm{AF})$, heart failure (HF), stroke, rheumatoid arthritis (RA), obesity, severe mental illness (SMI; schizophrenia, bipolar disorder and psychosis), learning disability, hypertension and asthma. In addition to the QOF indicators, and in recognition of the importance of chronic pain on occupational function, a chronic pain indicator ${ }^{20}$ was derived based on receipt of any prescriptions listed in British National Formulary medication chapters 4.7.2 or 4.7.3 (with repeat, repeat dispensed or automatic issue type).$^{20}{ }^{21}$ QOF conditions excluded from our analysis were: palliative care (patients are unlikely to be in employment), osteoporosis, dementia and chronic kidney disease (excluded due to the small numbers with these diagnoses in the age group under study). In addition to the count of long-term conditions, we included a binary covariate for QOF depression.

QOF measures have been shown to underestimate the prevalence of disorders in the general population due to the reliance on presentation to the GP for diagnosis. ${ }^{22}$ QOF diagnosis is therefore a specific but not sensitive measure ${ }^{23}$ with a low false-positive but high false-negative rates. 
Fit note (LDN)

Date of the first fit note was derived for each patient based on digital fit notes issued between 1 January 2014 and 30 April 2016. We excluded fit notes issued before this period because although digital fit notes were introduced in 2012, they were not in widespread use until 2014. Due to the lack of information on prior sickness certification (before January 2014), we could not determine whether a 'first fit note' during the study period represented the individual's first ever sickness certification.

\section{Symptoms on first fit note (LDN)}

There were a wide variety of conditions (recorded using Read codes) in the electronic GP record when a first fit note was issued. The creation of 11 groups was based on a pragmatic mix of the size of the groups, the way diagnoses are grouped in International Classification of Diseases Tenth Revision (ICD-10) and past fit note research. ${ }^{24-26}$ There were two mental health groups: (1) mental disorder, (2) 'stress' and specific external stressors (which includes bereavement and relationship breakdown), and eight non-mental health groups were: (1) musculoskeletal, (2) infection, (3) surgery, (4) other physical health condition, (5) fatigue, (6) obstetric presentations, (7) injury and (8) a group for 'other' conditions that could not easily be categorised (eg, administrative codes). The 'other physical health' group contained individual symptoms that could not be attributed to a single system or group of diagnoses.

\section{Mental healthcare indicator: antidepressants (LDN)}

Antidepressant prescription was measured in LDN as a binary variable indicating whether the patients had received an antidepressant prescription since 2006. This included antidepressants in the chapter 4.3 of the British National Formulary. ${ }^{21}$ A set of codes was used to extract antidepressants and to exclude medication used for other conditions, such as low dose tricyclic medication used for neuropathic pain. The list of excluded medications was previously defined by Schofield et al. ${ }^{27}$ We derived two outcomes related to treatment access: (1) received an antidepressant prescription in the year after receiving a first fit note and (2) ever received an antidepressant prescription at any time from 1993 to 2017.

\section{Mental healthcare indicator: psychological treatment (CRIS)}

Contact with IAPT psychological treatment was extracted from the CRIS dataset as a binary variable indicating whether individuals had attended an IAPT session. We derived two outcomes related to treatment access: (1) attended an IAPT session in the year after receiving their first fit note and (2) ever attended an IAPT session at any time from 2008 to 2017. Sessions included contact via both high and low intensity pathways and both phone and face to face contact.

Mental healthcare indicator: secondary care (CRIS)

Contact with secondary mental healthcare was measured as any contact with secondary mental healthcare services,

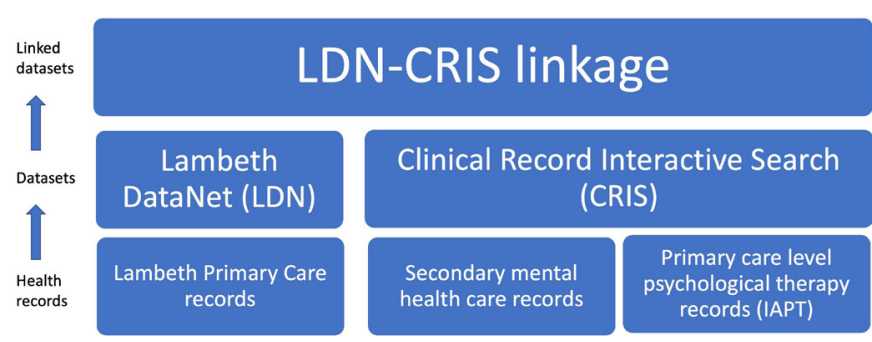

Figure 1 CRIS-LDN linkage.

measured as (1) having contact with secondary care services in the year after a first fit note; and (2) ever having contact with secondary care services. This could include many routes into care (including GP referral) and different mental health interventions delivered in secondary care. ${ }^{2829}$

\section{Data linkage}

This is longitudinal descriptive study using a record linkage between a primary care database and a secondary care mental health database in Lambeth (figure 1). We analysed the records of patients registered for 28 months, from 1 January 2014 to 30 April 2016. LDN data were extracted in May 2017 from the primary care clinical record system, EMIS Web. We restricted the sample to working age adults (aged 16-60 years) to prevent exit from the workforce due to reaching statutory pension age (60-65 years during our study period), as retirement status was not captured in the health records. The record linkage was achieved using deterministic methods based on an exact match on the pseudonymised NHS number. LDN NHS numbers were scrambled using a oneway encryption process using 'open source pseudonymiser' software. For linkage, the Clinical Data Linkage Service used exactly the same process to encrypt local NHS numbers joined to the CRIS pseudonym. CRIS and LDN cases were then joined within the secure safe haven on the now shared encrypted NHS number, following project-specific approvals. The project-specific extract was fully anonymised by replacing the pseudonymised unique identifier with a project-specific anonym before access was granted to the researchers. SLaM provides services across four London boroughs, so we expected a roughly $25 \%$ match rate (the proportion of people in CRIS who are registered with a Lambeth GP). The LDNCRIS linkage match rate was $27.8 \%$.

\section{Statistical analysis}

Baseline characteristics among study patients and rates of contact with mental healthcare were described with counts and percentages. Binary logistic regression models were used to assess associations between demographic variables, condition at first fit note and contact with mental healthcare. To take account of clustering of patients with GP practices, SEs were adjusted with a sandwich estimator. ${ }^{30}$ To understand the influence of fit note receipt on mental health treatment access, we conducted these analyses in three subsamples: (1) patients receiving 
a first fit note between 1 January 2014 and 30 April 2016; (2) patients with a condition recorded at first fit note; (3) patients registered with a GP in the Borough of Lambeth 1 January 2014 and 30 April 2016. The mean number of fit notes received per person within the 28-month period was analysed by demographic variables. All models were adjusted for covariates previously shown to be associated with fit note receipt, ${ }^{12}$ including demographic variables (age, gender and LSOA deprivation) and chronic disorders (such as depression and cardiovascular conditions). For all analyses, we included each patients' first fit note during the study period (representing 28\% of all fit notes, all other fit notes were excluded). All models were estimated using Stata V.15 (Stata Corporation, USA).

\section{Ethical committee approval}

CRIS was established in 2008 and approved by the Oxfordshire Research Ethics Committee (reference 18/ SC/0372) (https://doi.org/10.1186/1471-244X-9-51). ${ }^{28}$ Approval for linkage with LDN was granted by Lambeth Clinical Commissioning Group and Information Governance Steering Group. This project was approved by the CRIS Oversight Committee in 2015.

\section{Patient and public involvement statement}

This work will continue to be disseminated to local community groups, such as Black Thrive Lambeth, healthcare providers, including South London and Maudsley NHS Trust, and Lambeth Datanet Steering Group.

\section{RESULTS}

The analytical sample included 293933 people, after removing 69507 individuals with missing information on ethnicity $(10 \% \mathrm{n}=36530)$, deprivation $(6 \% \mathrm{n}=21878)$ or both $(3 \% \mathrm{n}=11$ 099). Compared with the analytical sample, excluded individuals (online supplemental table 1 ) were younger (18\% vs 3\% aged $16-20$ years), more likely to be men ( $56 \%$ vs $47 \%$ ), slightly less likely to report at least one type of long-term condition (29\% vs $30 \%$ ) and less likely to have received a fit note $(11 \%$ vs $14 \%)$.

\section{Demographic associations for access to mental healthcare indicators in the year after first fit note}

The distribution of mental healthcare indicators following a first fit note (for any reason - including mental or physical illness) is shown in table 1. A total of 32233 (11\%) received a fit note between 1 January 2014 and 30 April 2016. In the year after first fit note, $6288(20 \%)$ received an antidepressant prescription, $3179(10 \%)$ had an IAPT session, $2785(9 \%)$ had a secondary care contact and $23082(72 \%)$ had no contact with mental health services. In logistic regression models adjusted for long-term physical health conditions and mutually adjusted for demographic factors, minority ethnic groups were least likely to access any treatment for mental health after first fit note. The strongest association with no access to mental healthcare indicators in the year after first fit note was among those in the Black African group. There was no difference in contact with treatment overall by gender, but men and women accessed different forms of care. Women were more likely to access primary care services (antidepressant prescription/IAPT contact). Men and persons aged 16-30years were more likely to access secondary care. There was no association between deprivation and contact with mental healthcare in the year after first fit note and no association between IAPT contact and longterm conditions. Mental healthcare indicators were lowest in people with no health conditions, no fit notes and no depression diagnosis. Demographic variation in access to mental healthcare indicators in the year after first fit note was similar to demographic variation in the whole population (online supplemental table 2).

\section{Mental healthcare indicators by diagnosis on first fit note}

Among patients receiving a fit note during the study period, other physical health conditions at first fit note were the most prevalent $(31 \%)$, followed by musculoskeletal conditions (21\%) and then infection (17\%) (table 2). Fourteen per cent of people were noted by the GP to have a mental health presentation at the time of their first fit note, the majority being common mental disorders. Fiftynine per cent of patients with common mental disorder on the fit note were prescribed antidepressants in the year after first fit note, 33\% had contact with IAPT and $20 \%$ had contact with secondary mental healthcare. A quarter of people with common mental disorder had no contact with mental healthcare indicator in the year after first fit note.

Five per cent of fit notes were for stress, of which $25 \%$ were prescribed antidepressants, $22 \%$ had contact with IAPT and $7 \%$ had contact with secondary care. Sixty per cent had no mental healthcare indicator. Antidepressant prescriptions and IAPT contact in year after first fit note were highest among patients presenting with common mental disorder (59\% and 33\%). Secondary care contact was highest among patients with other mental health presentations $(76 \%)$ and drug and alcohol use disorders $(63 \%)$.

\section{Mental healthcare indicators in the year after first fit note for mental health condition or stress}

We found a higher prevalence of mental health conditions at first fit note among men, younger adults, people in the White British and the Mixed groups, amongst patients with multiple fit notes and multiple long-term conditions (table 3). Stress recorded at first fit note was less common (5\% compared with $14 \%$ of fit notes for mental health), it was highest among women, in the Black Caribbean group, among people with more long-term conditions, and people with more fit notes.

No access to mental healthcare in the year after mental health on first fit note was highest among people in Black African and Black Caribbean groups and people with fewer fit notes. 


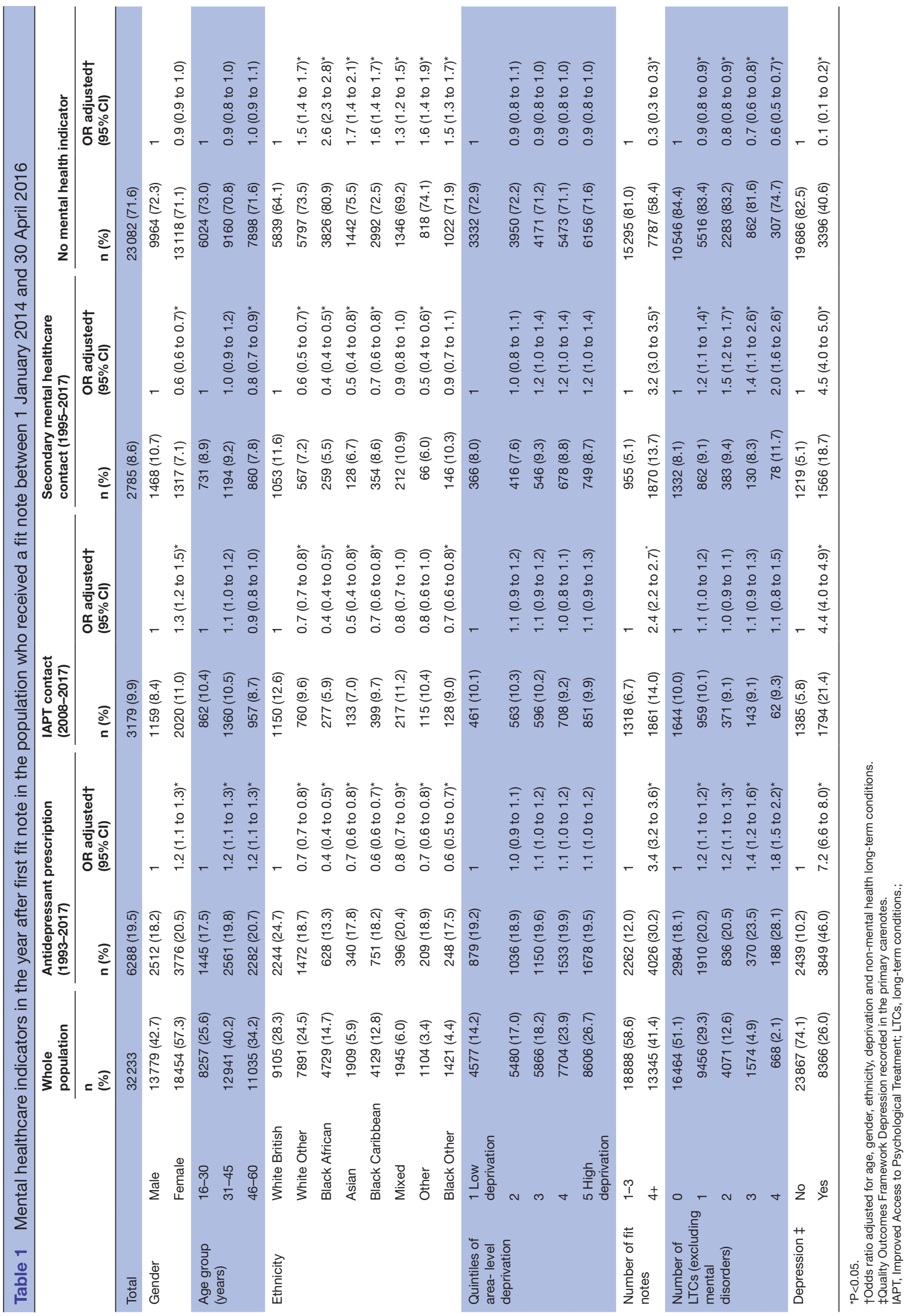




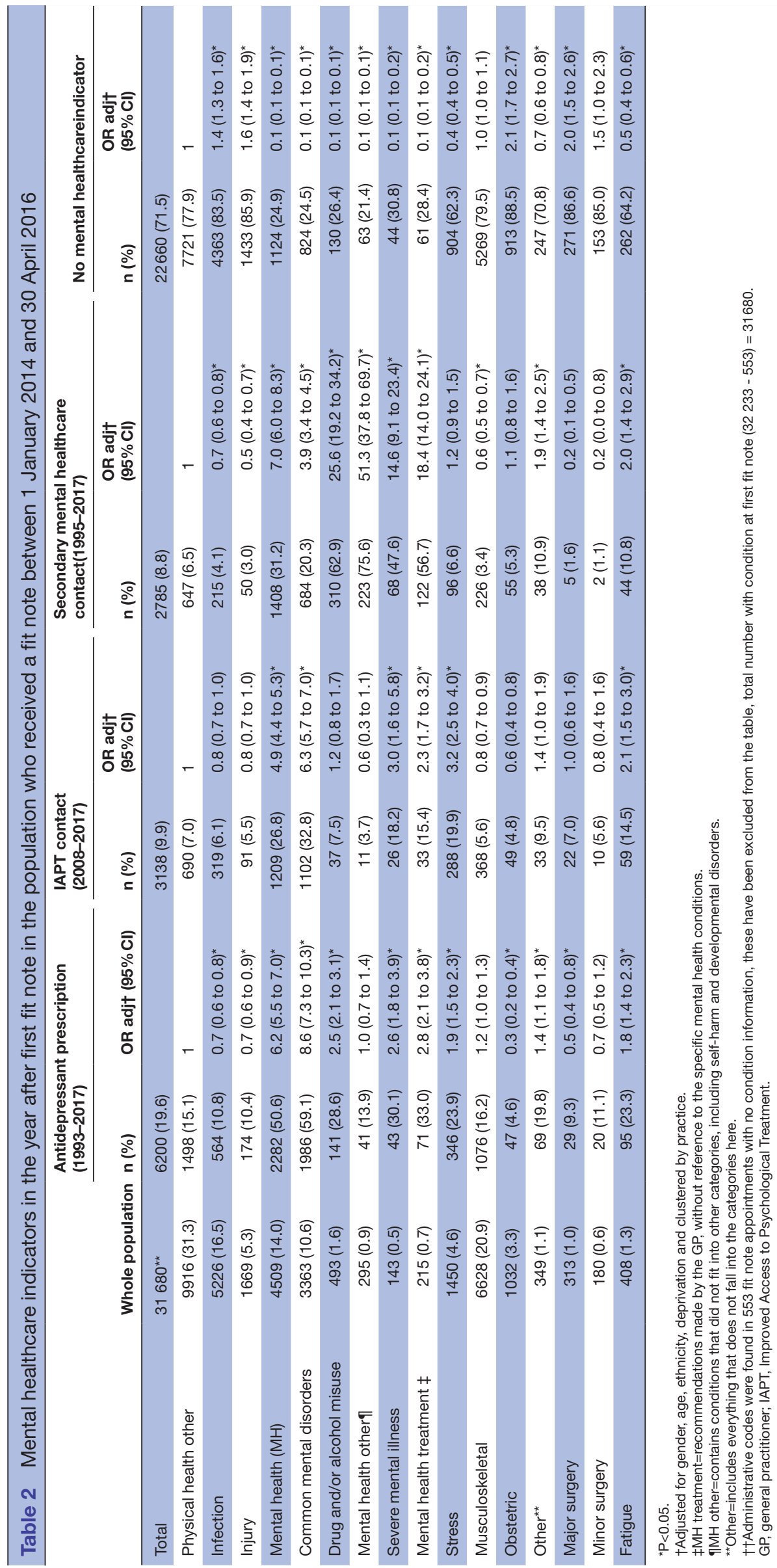


Table 3 Mental healthcare indicators in the year after first fit note for mental health condition or stress

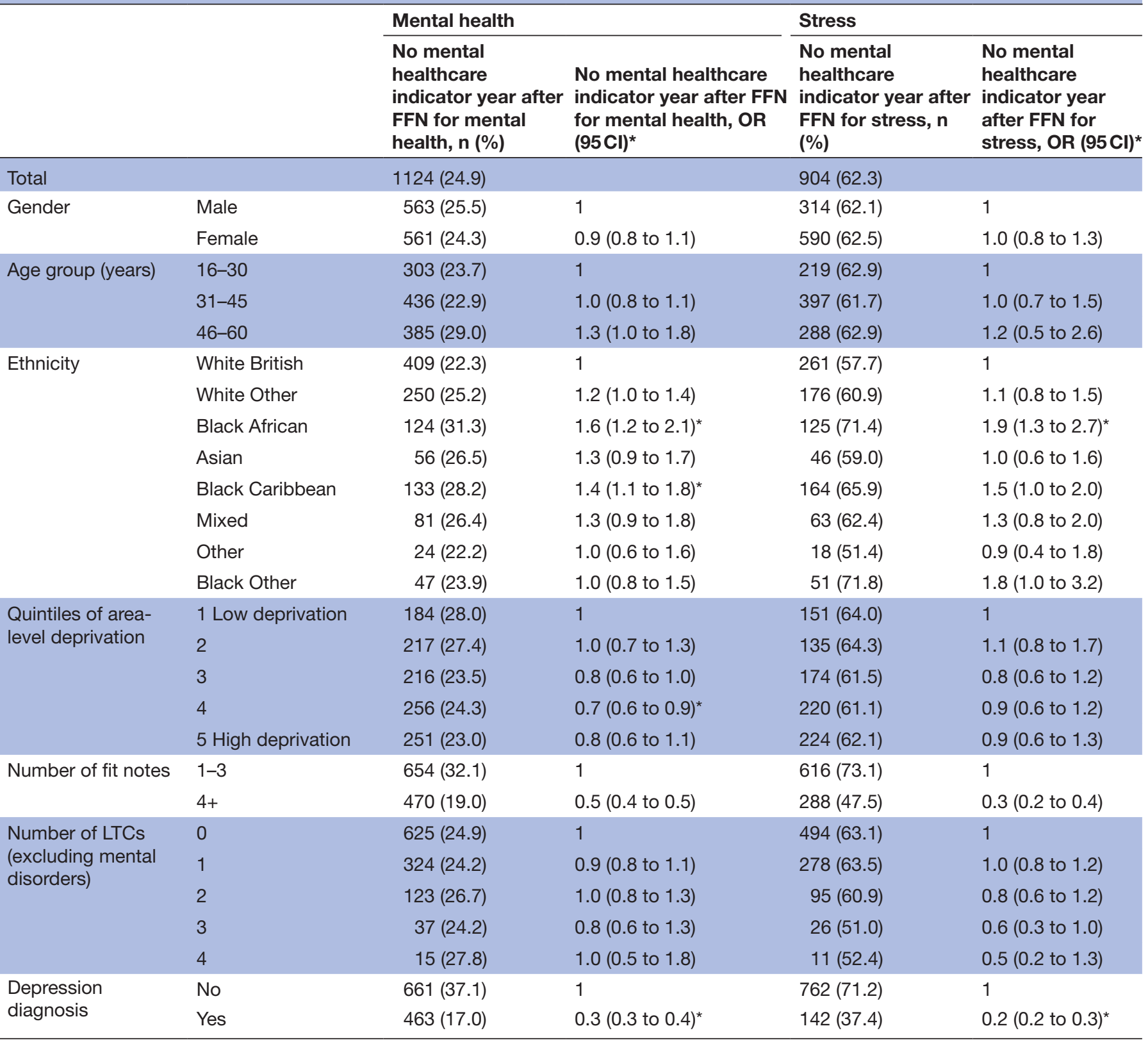

${ }^{*}$ Odds ratio mutually controlled for gender, age, ethnicity, deprivation and clustered by practice.

FFN, first fit note; LTC, long-term condition.

\section{DISCUSSION}

Our data suggest that around $72 \%$ of people who have a mental disorder recognised by the GP at the point of first fit note have a mental healthcare indicator in the year after first fit note. Not surprisingly, people receiving fit notes where the consultation focused on a physical disorder reported a much lower likelihood of receiving mental health interventions or care in the next year. In people where the GP had recognised stress in some form or other, the majority received no specific mental health intervention.

Our findings suggest that people with mental disorders, when recognised by the GP, usually do receive some form of mental health intervention following receipt of fit notes. It is possible (with some caveats discussed further) that these numbers could be improved, but the coverage is higher than we were expecting based on previous literature. ${ }^{31-33}$ Evidence suggests that in people receiving fit notes for a physical disorder, particularly chronic or recurrent conditions like musculoskeletal disorders, there is a high level of comorbidity with mental disorders that is frequently unrecognised ${ }^{34-36}$ Certain physical health conditions known to have high levels of comorbidity with mental disorders, for example, musculoskeletal disorders and stress-related reasons for sickness absence, could routinely be the focus of a mental health assessment, and where appropriate, mental health intervention at the point of fit note. 
An important caveat is that our indicators of mental health intervention are crude. We chose the three indicators (antidepressant prescription, IAPT contact and secondary care contact) because they are readily measured and common; however, they do not describe the breadth of interventions available, and it would be misleading to suggest that an individual who does not receive any of these interventions necessarily is not receiving any form of mental healthcare; therefore, our estimates of treatment are likely to be an underestimate. Medications for severe mental illness are typically initiated in secondary care and the number of people who do not have secondary care contact who get a prescription from primary care is likely to be small; however, this could usefully be explored in follow-up studies.

Furthermore, we do not know whether the treatments received were necessarily appropriate. While there is concern that people with mental disorders do not receive adequate treatment with interventions that could be beneficial, ${ }^{37-39}$ there is also concern that antidepressants are too widely prescribed, ${ }^{40}{ }^{41}$ and we do not know from the data shown here, whether the antidepressant prescription was appropriate and of sufficient duration.

Another important issue our data have uncovered is differential access to mental health interventions and care for different demographic groups. Having no mental healthcare indicator after first fit note for a mental health condition was most common among people in the Black African and Black Caribbean groups (table 3). Differences in access to mental healthcare by ethnicity have been described in the UK; ethnic minority groups are less likely to access appropriate treatment. ${ }^{29} 4243$ People from Black ethnic groups in London have been found to be less likely to be diagnosed with depression in primary care. ${ }^{27} 44$ Compared with White British and other ethnic groups, Black Caribbean and Black African groups are more often referred to secondary care when recognised to have a mental health problem by their GP, rather than being treated in primary care, and are more likely to experience coercive pathways into mental healthcare. ${ }^{29} 43$ Structural inequalities lead to delays in the identification and treatment of mental health problems. ${ }^{43}$ Difficulty accessing healthcare is likely to be compounded by insecure and inflexible employment; working-age adults from black ethnic groups are twice as likely to be in insecure jobs as White peers and have less access to sick pay. ${ }^{45}$

Population level data about workplace environments suggests those most at risk of long-term health conditions are least likely to receive occupational health support. ${ }^{46}$ Intersectional approaches and studies including information on employment type are needed in future health service use research to examine and improve the mental healthcare trajectories of working age adults. ${ }^{44} 47-49$

\section{Limitations}

While our use of complete population data at an individual level (rather than aggregated) improves on previous work in this field, there are inevitable limitations in using routine data, such as completeness of data registration. We focused on a single geographical area for which we had GP data. This had the advantage of providing information on virtually all individuals seeking help within that population but cannot be assumed to be representative of other UK populations, and our findings should be tested in other areas. We were unable to explore contextual factors such as the nature of employment, educational level or benefit information, making it impossible to ascertain whether fit notes were issued for individuals in work as opposed to those already out of work who were applying for health benefits, and we were limited to using area level deprivation scores. We could not determine the proportion of people who did not receive a fit note despite being ill, either due to relaxed work policies or because they feel unable to take time off work. Furthermore, long-term health conditions were assessed using QOF, which provides incomplete diagnostic information. Our mental health indicators were limited to antidepressants, IAPT and secondary care contact, and therefore, our estimates of treatment are likely to be an underestimate. We were unable to access information on the reason for sickness certification written on the fit note because free-text data extraction is not permitted from anonymised primary care datasets. The study population are registered at a Lambeth practice so it is unlikely that they are receiving mental health primary care treatment out of area, although we cannot rule this out. Further studies are needed to explore the length of contact with mental healthcare, treatment adherence and clinical outcomes.

\section{Summary}

Our findings suggest that people with mental disorders, when recognised by the GP, usually do get some form of mental health intervention following receipt of fit notes;however, Black African and Black Caribbean groups have less complete access to mental healthcare in the year after a fit note for a mental health condition, compared to the White British group. Further research is needed to examine whether the fit note increases access to care in groups at highest risk of LTSA and to reduce the barriers that prevent equal access to mental healthcare at first fit note.

Twitter Ewan Carr @ewancarr

Acknowledgements The authors acknowledge the assistance of Amelia Jewell and Megan Pritchard in creating the data linkage, and to Dr Catherine Polling for advising on the categorisation of read codes into groups and subgroups.

Contributors SD designed the study, analysed the data and drafted the manuscript. MH, SH and IM advised extensively throughout the process. EC advised on the statistical analysis. MB, MA and SS provided expertise and guidance on extracting, analysing and interpreting the data. All authors commented on the final manuscript. All authors meet the ICMJE criteria for authorship. 
Funding This paper represents independent research funded by a part funded by the Royal College of Psychiatrists' Donald Dean Fellowship (award number not applicable) and the National Institute for Health Research Biomedical Research Centre at South London and Maudsley NHS Foundation Trust and King's College London (IS-BRC-1215-20018)

Disclaimer The views expressed are those of the authors and not necessarily those of the Royal College of Psychiatrists, the National Health Service, theNational Institute for Health Research or the Department of Health and Social Care.

Competing interests $\mathrm{MH}$ receives funding from Janssen as part of the RADARCNS consortium which includes a project on depression. He is a principal investigator of RADAR-CNS, a precompetitive public-private partnership cofunded by Innovative Medicines Initiative (European Commission) and European Federation of Pharmaceutical Industries and Associations. He has also been an independent expert witness in group litigations instructed by claimants against pharmaceutical companies for alleged harmful effects of their products.

Patient consent for publication Not required.

Provenance and peer review Not commissioned; externally peer reviewed.

Data availability statement Data may be obtained from a third party and are not publicly available. Access to the deidentified data in this study can be applied for through Lambeth Datanet and South London and Maudsley Biomedical Research Centre (BRC), De Crespigny Park, London SE5 8AF (cris.administrator@slam.nhs. uk). Access is granted only if approval is given by both the BRC and the Lambeth Datanet Steering Group.

Supplemental material This content has been supplied by the author(s). It has not been vetted by BMJ Publishing Group Limited (BMJ) and may not have been peer-reviewed. Any opinions or recommendations discussed are solely those of the author(s) and are not endorsed by BMJ. BMJ disclaims all liability and responsibility arising from any reliance placed on the content. Where the content includes any translated material, BMJ does not warrant the accuracy and reliability of the translations (including but not limited to local regulations, clinical guidelines, terminology, drug names and drug dosages), and is not responsible for any error and/or omissions arising from translation and adaptation or otherwise.

Open access This is an open access article distributed in accordance with the Creative Commons Attribution Non Commercial (CC BY-NC 4.0) license, which permits others to distribute, remix, adapt, build upon this work non-commercially, and license their derivative works on different terms, provided the original work is properly cited, appropriate credit is given, any changes made indicated, and the use is non-commercial. See: http://creativecommons.org/licenses/by-nc/4.0/.

\section{ORCID iDs}

Sarah Dorrington http://orcid.org/0000-0002-6462-1880

Ewan Carr http://orcid.org/0000-0002-1146-4922

Mark Ashworth http://orcid.org/0000-0001-6514-9904

\section{REFERENCES}

1 Henderson M, Harvey SB, Øverland S, et al. Work and common psychiatric disorders. J R Soc Med 2011;104:198-207.

2 Gabbay M, Shiels C, Hillage J. Sickness certification for common mental disorders and GP return-to-work advice. Prim Health Care Res Dev 2016;17:437-47.

3 Department for Work and Pensions. Department of work and pensions online statistics: Stat-Xplore. Department for Work and Pensions, Department of Health, 2020.

4 Health and Safety Executive. Health and safety at work: summary statistics for great Britain 2019. Health and Safety Executive, 2019.

5 Waddell G. Preventing incapacity in people with musculoskeletal disorders. Br Med Bull 2006;77-78:55-69.

6 Harvey SB, Henderson M, Lelliott P, et al. Mental health and employment: much work still to be done. Br J Psychiatry 2009;194:201-3.

7 Black C. Dame Carol Black's Review of the health of Britain's working age population : working for a healthier tomorrow. London: TSO, 2008.

8 Thornicroft G, Chatterji S, Evans-Lacko S, et al. Undertreatment of people with major depressive disorder in 21 countries. $\mathrm{Br} \mathrm{J}$ Psychiatry 2017;210:119-24.

9 Davidson JR, Meltzer-Brody SE. The underrecognition and undertreatment of depression: what is the breadth and depth of the problem? J Clin Psychiatry 1999;60.
10 Alonso J, Liu Z, Evans-Lacko S, et al. Treatment gap for anxiety disorders is global: results of the world mental health surveys in 21 countries. Depress Anxiety 2018;35:195-208.

11 McManus S, Bebbington PE, Jenkins R, et al. Data resource profile: adult psychiatric morbidity survey (APMS). Int J Epidemiol 2020;49:361-2.

12 Dorrington S, Carr E, Stevelink SAM, et al. Demographic variation in fit note receipt and long-term conditions in South London. Occup Environ Med 2020;77:418-26.

13 Hatch SL, Frissa S, Verdecchia M, et al. Identifying sociodemographic and socioeconomic determinants of health inequalities in a diverse London community: the South East London community health (SELCoH) study. BMC Public Health 2011;11:861.

14 Lambeth Council. Lambeth demography Factsheet, 2017. Available: https://www.lambeth.gov.uk/sites/default/files/ssh-demographyfactsheet-2017.pdf2020

15 Lambeth Council. State of the borough. Lambeth Council, 2016.

16 Trust for London. Overview of London boroughs. Trust for London: Tackling poverty and inequality, 2019. https://www.trustforlondon. org.uk/data/boroughs/overview-of-london-boroughs/

17 Stewart R, Soremekun M, Perera G, et al. The South London and Maudsley NHS Foundation trust biomedical research centre (SLAM $\mathrm{BRC}$ ) case register: development and descriptive data. BMC Psychiatry 2009;9:1-12

18 Greater London Authority. LSOA Atlas, 2014. Greater London authority. Available: https://data.london.gov.uk/dataset/lsoa-atlas

19 Department of Health. QOF Business Rules - version 35. Department of Health, 2017.

20 GSTT Charity. From One to Many: Exploring people's progression to multiple long-term conditions in an urban environment, 2020. Available: https://www.gsttcharity.org.uk/what-we-do/ourprogrammes/multiple-long-term-conditions/one-many-exploringpeoples-progression2018

21 National Institute for Health and Care Excellence. British National formulary (BNF), 2020. Available: https://bnf.nice.org.uk/2020

22 The Kings Fund. Impact of quality and outcomes framework on health inequalities. The Kings Fund, 2011.

23 Economou A, Grey M, McGregor J, et al. The health informatics cohort enhancement project (HICE): using routinely collected primary care data to identify people with a lifetime diagnosis of psychotic disorder. BMC Res Notes 2012;5:95.

24 Shiels C, Gabbay MB, Hillage J. Change in diagnosis on fit notes within an episode of certified sickness absence. Fam Pract 2016;33:510-6.

25 Starzmann K, Hjerpe P, Dalemo S, et al. Diagnoses have the greatest impact on variation in sick-leave certification rate among primarycare patients in Sweden: a multilevel analysis including patient, physician and primary health-care centre levels. Scand J Public Health 2015;43:704-12.

26 Shiels C, Gabbay M, Hillage J. Factors associated with prevalence and types of 'may be fit' advice on fit notes: a cross-sectional primary care analysis. Br J Gen Pract 2014;64:e137-43.

27 Schofield P, Das-Munshi J, Mathur R, et al. Does depression diagnosis and antidepressant prescribing vary by location? analysis of ethnic density associations using a large primary-care dataset. Psychol Med 2016;46:1321-9.

28 Stewart R, Soremekun M, Perera G, et al. The South London and Maudsley NHS Foundation trust biomedical research centre (SLAM $\mathrm{BRC}$ ) case register: development and descriptive data. $B M C$ Psychiatry 2009;9:51.

29 Chui Z, Gazard B, MacCrimmon S, et al. Inequalities in referral pathways for young people accessing secondary mental health services in South East London. Eur Child Adolesc Psychiatry 2021;30:1113-28.

30 StataCorp. Stata survey data reference manual: svy, 2013. Available: https://www.stata.com/manuals13/svy.pdf

31 Kendrick T, Dowrick C, McBride A, et al. Management of depression in UK general practice in relation to scores on depression severity questionnaires: analysis of medical record data. BMJ 2009;338:b750.

32 Evans-Lacko S, Aguilar-Gaxiola S, Al-Hamzawi A, et al. SocioEconomic variations in the mental health treatment gap for people with anxiety, mood, and substance use disorders: results from the who world mental health $(\mathrm{WMH})$ surveys. Psychol Med 2018;48:1560-71.

33 Patel V, Maj M, Flisher AJ, et al. Reducing the treatment gap for mental disorders: a WPA survey. World Psychiatry 2010;9:169-76.

34 Knudsen AK, Øverland S, Aakvaag HF, et al. Common mental disorders and disability pension Award: seven year follow-up of the husk study. J Psychosom Res 2010;69:59-67. 
35 Mykletun A, Overland S, Dahl AA, et al. A population-based cohort study of the effect of common mental disorders on disability pension awards. Am J Psychiatry 2006;163:1412-8.

36 Mykletun A, Heradstveit O, Eriksen K, et al. Health anxiety and disability pension Award: the husk study. Psychosom Med 2009;71:353-60.

37 Department of Health. Closing the gap: priorities for essential change in mental health. Department of Health, 2014.

38 NHS England. Achieving better access to mental health services by 2020. England: Department of Health, NHS, 2014

39 Salaheddin K, Mason B. Identifying barriers to mental health helpseeking among young adults in the UK: a cross-sectional survey. $\mathrm{Br}$ J Gen Pract 2016;66:e686-92.

40 Spence D. Are antidepressants overprescribed? Yes. BMJ 2013;346:f191.

41 Johnson CF, Williams B, MacGillivray SA, et al. 'Doing the right thing': factors influencing GP prescribing of antidepressants and prescribed doses. BMC Fam Pract 2017;18:72.

42 Brown JSL, Ferner H, Wingrove J, et al. How equitable are psychological therapy services in South East London now? A comparison of referrals to a new psychological therapy service with participants in a psychiatric morbidity survey in the same London borough. Soc Psychiatry Psychiatr Epidemiol 2014;49:1893-902.

43 Morgan C, Mallett R, Hutchinson G, et al. Negative pathways to psychiatric care and ethnicity: the bridge between social science and psychiatry. Soc Sci Med 2004;58:739-52.

44 Bhui K, Stansfeld S, Hull S, et al. Ethnic variations in pathways to and use of specialist mental health services in the UK. systematic review. Br J Psychiatry 2003;182:105-16.

45 Congress TU. Covid-19 and insecure work, 2021.

46 Raynal A, Hermanns R, Robson S, et al. Everybody should have access to occupational health services. BMJ 2019;364:k5220.

47 Pearlin LI, Schieman S, Fazio EM, et al. Stress, health, and the life course: some conceptual perspectives. $J$ Health Soc Behav 2005:46:205-19.

48 Hatch SL, Dohrenwend BP. Distribution of traumatic and other stressful life events by race/ethnicity, gender, Ses and age: a review of the research. Am J Community Psychol 2007;40:313-32.

49 Gazard B, Chui Z, Harber-Aschan L, et al. Barrier or stressor? the role of discrimination experiences in health service use. BMC Public Health 2018;18:1354. 